\title{
LA NEGOCIACIÓN COLECTIVA COMO INSTRUMENTO PARA FRENAR LOS DAÑOS A LA SALUD LABORAL DERIVADOS DE LA PRECARIEDAD
}

\author{
Collective bargaining as instrument to stop damage to occupational health \\ from job insecurity
}

Alfonso Ríos VeladA*

Responsable de Salud Laboral, CCOO de Euskadi

\section{RESUMEN}

La precariedad laboral, característica de nuestro mercado de trabajo, es una realidad multifactorial que tiene incidencia en las malas condiciones de trabajo y en el deterioro de la salud de las personas trabajadoras. En este contexto, la interacción entre los agentes sociales a través de la negociación colectiva es, con el Derecho del Trabajo, la herramienta de las personas trabajadoras para tener unas condiciones de trabajo dignas, así como para mejorarlas.

En este sentido, existe margen para que en la negociación colectiva se aborden materias que defiendan la salud en el trabajo, teniendo en cuenta que las medidas que contribuyen a combatir situaciones de precariedad laboral tienen incidencia también en la salud de las personas. La mayor garantía para un buen resultado en la negociación colectiva es que abarque el ámbito más amplio posible, generando derechos colectivos para el mayor número de personas.

Palabras clave: precariedad laboral, negociación colectiva, salud laboral, convenio colectivo.

\section{ABSTRACT}

Job insecurity, as a feature of our labour market, is a multifactorial fact that impacts on poor working conditions and harms workers' health. In this context, the interaction between social partners through collective bargaining, along with Labour Law, is the tool that working people have to get decent working conditions, and also to improve them.

In this respect, there is room for collective bargaining to deal with matters to uphold occupational health, considering that measures that fight precariousness of work have also impact on people's health. The greatest guarantee for a good result on collective bargaining is to have a wider scope, to create collective rights for the greatest possible number of people.

Keywords: job insecurity, collective bargaining, occupational health, collective agreement.

* Correspondencia a: Alfonso Ríos Velada. C/ Uribitarte 4, Bilbao (Bizkaia), España. - alfonsorios@ccoo.eus

Cómo citar: Ríos Velada, Alfonso. (2020). «La negociación colectiva como instrumento para frenar los daños a la salud laboral derivados de la precariedad"; Lan Harremanak, 44, 218-237. (https://doi.org/10.1387/lan-harremanak.22213).

Recibido: 05 noviembre, 2020; aceptado: 07 diciembre, 2020.

ISSN 1575-7048 - elSSN 2444-5819 / (c) 2020 UPV/EHU 


\section{Introducción}

Los daños a la salud que se derivan de las condiciones laborales están presentes en los puestos de trabajo. Los accidentes laborales son la parte más visible y dramática de las consecuencias que el trabajo tiene en la salud. Existen también las enfermedades profesionales, más difíciles de detectar debido a que no son visibles en el momento en que se están contrayendo, y las patologías derivadas de los riesgos psicosociales en el trabajo, generando ambas igualmente perjuicios entre las personas trabajadoras.

Se necesitan mecanismos para reducir y eliminar estos riesgos. Dentro de las técnicas y la práctica que la prevención de riesgos laborales ofrece, y más allá de lo que el derecho del trabajo estipula, la negociación colectiva amplía las posibilidades de minimizar e incluso reducir los daños a la salud derivados del trabajo, por lo que es necesario extender este objetivo en el ámbito de la propia negociación colectiva.

En la primera parte del artículo se analiza cómo la precariedad laboral está detrás de muchos daños a la salud que tienen que ver con la exposición a determinadas condiciones de trabajo. En su primera sección se realiza un resumen de lo que es la precariedad laboral, para pasar en la segunda sección a mostrar las consecuencias concretas de la precariedad en la salud laboral. La tercera sección, por último, se centra en la especial incidencia que una práctica empresarial concreta, como es la subcontratación, tiene en la generación de los daños a la salud.

La segunda parte muestra las directrices generales que desde la negociación colectiva se pueden abordar para la defensa de la salud en el trabajo. En una primera sección se resume lo que es la negociación colectiva, como instrumento eficaz para mejorar las condiciones de trabajo y los derechos laborales. La segunda sección se centra en las cláusulas relativas a la participación de las personas trabajadoras en salud laboral en la empresa. La tercera sección aborda las cláusulas útiles para limitar las interferencias de las mutuas en los procesos de recuperación de las personas que puedan estar en proceso de recuperación de una dolencia concreta. Y la cuarta sección, por último, muestra la necesidad de abrir todas estas cláusulas a colectivos aún ajenos a la negociación colectiva, como son las personas que trabajan como falsas autónomas y las personas prestadoras de servicios en plataformas digitales.

La negociación colectiva se entiende como un mecanismo más para la defensa de unas condiciones de trabajo dignas, y se usa por lo tanto con el objetivo de eliminar los accidentes de trabajo, las enfermedades profesionales y todos los daños que perturban el bienestar de la clase trabajadora. 


\section{Precariedad laboral e incidencia en la salud de las personas}

\subsection{La precariedad laboral: fenómeno multifactorial}

Las personas trabajadoras viven en un mercado laboral cambiante en el cual se dan nuevas situaciones y nuevas realidades que exigen una constante adaptación. En ese contexto general, la precariedad laboral no es un fenómeno nuevo, sino que es algo que ha arraigado, y que se ceba con unos determinados segmentos de la población trabajadora, como las personas jóvenes y las mujeres.

La precariedad laboral es un fenómeno multifactorial, no presenta una única cara ni una sola característica. Se entrecruzan los bajos salarios, la temporalidad en la contratación o la contratación a tiempo parcial no deseada, características todas ellas que acaban desembocando en la incertidumbre sobre el futuro y la continuidad de la relación laboral. La denominada brecha salarial, que afecta en su parte negativa a las mujeres trabajadoras, es un fenómeno que también se relaciona de forma directa con la precariedad laboral.

Todas las situaciones descritas degradan el trabajo, lo que incide en otro tipo de situaciones, como la falta de reconocimiento profesional. No obstante, las situaciones descritas también pueden afectar a personas trabajadoras que tengan un contrato indefinido. La inclusión de elementos de flexibilidad que son exclusivamente gobernados y dirigidos por la empresa, y que alteran de forma arbitraria algunas condiciones de trabajo, como el horario o la movilidad geográfica, generan incertidumbre, no tanto sobre la continuidad de la relación laboral, pero si sobre la forma en la que se presta el trabajo.

En la discontinuidad de las relaciones laborales influye también la «inestabilidad de las empresas, que nacen, mueren, se reproducen, se segregan, desaparecen y se trasladan sin apenas trabas» (CCOO, 2007: 348). Precariedad e incertidumbre en torno al futuro de la relación laboral vienen afectadas por un número significativo de factores, lo que ha contribuido a su extensión en el mercado de trabajo.

La temporalidad en la contratación es la forma en la que en el conjunto del estado se ha venido creando empleo pero, obviamente, no un empleo de calidad. Este modelo de contratación se incrementa a finales de la década de los 80 , y mantiene los niveles alcanzados en esta época durante los 90 y la primera mitad de los 2000. A partir de 2007 ni siquiera es posible hablar de creación de empleo, puesto que desde comienzos de 2008 hasta finales de 2013, y según el estudio elaborado por Lorente y Guamán (2018: 50), se destruyeron 3 millones setecientos mil empleos, o lo que es lo mismo, un $17^{\prime} 8 \%$ de lo que era el empleo total del conjunto del estado. Y es que «la economía española es, dentro de la UE, la que más empleo crea en las expansiones económicas, y la que más empleo destruye en las recesiones» (Lorente y Guamán., 2018: 50). No obstante, el empleo creado en épocas de expansión económica está alejado del empleo de calidad, al expandirse la temporalidad en la contratación. 
La incertidumbre en relación al futuro de la relación laboral, y por lo tanto a la percepción de poder mantener unos ingresos estables o continuados, afecta a los proyectos de vida futura de las personas que tienen un contrato temporal. Por otro lado, también hay situaciones de precariedad laboral que se entrelazan con los modos de vida de nuestras sociedades y contribuyen a perpetuarlos, como es el caso del trabajo a tiempo parcial. Es un fenómeno que afecta de forma mayoritaria a las mujeres trabajadoras, y que se relaciona con la doble presencia y con la segregación que se da en el trabajo doméstico y en el ámbito de los cuidados (Fina, 2001: 88). Precariedad laboral y otras esferas de la precariedad vital se presentan de forma asociada.

Asimismo, el empleo a tiempo parcial, en especial para las personas jóvenes que acceden al mercado de trabajo, viene relacionada en muchas ocasiones con la existencia de otros ingresos que se derivan del núcleo familiar y de la convivencia en el mismo. No se puede afirmar en estos casos que se esté potenciando un proyecto de vida independiente más allá del núcleo familiar.

Todas las realidades descritas son las que están definiendo en el presente la precariedad laboral. Estas realidades, como se explicará a continuación, deterioran la salud de las personas en sus puestos de trabajo y fomentan accidentes de trabajo y enfermedades que se derivan de las condiciones laborales.

\subsection{Precariedad laboral y daños a la salud en el trabajo}

Una persona que accede a un puesto de trabajo con un contrato temporal, sobre todo si es una persona joven que atraviesa sus primeras experiencias en el mercado laboral, accede con un déficit en la experiencia sobre cómo prevenir los riesgos en el puesto, por lo menos en comparación con personas que hayan estado más tiempo en ese puesto.

Son las propias instituciones las que reconocen este hecho. En una entrevista publicada en el diario Deia el 3 de julio de $2017^{1}$ el entonces director de Osalan, el Instituto Vasco de Seguridad y Salud en el Trabajo, Alberto Alonso, afirmaba que en torno al 30\% de los accidentes de trabajo que se producen en la CAV ocurren durante el primer año en un puesto de trabajo, calificándose como crítico el primer mes. En el momento de la publicación de la entrevista, y según se afirma igualmente en ella, el $60 \%$ de los contratos laborales que se firmaban en Euskadi duraban menos de un mes.

Si hay empresas contratando a personas por menos de un mes (en periodos que duran por ejemplo veinte días, pero también tres o incluso uno), se constata un hecho que no incentiva a la formación de las personas trabajadoras. En

\footnotetext{
${ }^{1}$ La entrevista completa está disponible en https://www.deia.eus/economia/2017/07/03/alberto-alonso-30-accidentes-dan/585628.html (Accedido: 4-12-2020)
} 
este caso no existe una capacitación real que permita prevenir los riesgos a la salud en el trabajo, pese a tratarse de una obligación que tiene que cumplir la empresa dentro de la jornada laboral, o si no es posible, descontando de la jornada el tiempo invertido en la formación, tal y como viene enunciado en el artículo 19 de la Ley 31/1995, de 8 de noviembre, de Prevención de Riesgos Laborales.

Las empresas que realizan contratos laborales por tan pocos días no emplean tiempo alguno en organizar esta formación, y es posible que incluso lo consideren «malgastar» el poco tiempo que la persona trabajadora va a estar desempeñando su tarea. Es en cualquier caso un incumplimiento empresarial que tiene consecuencias en términos de aumento de la siniestralidad laboral.

Falta de experiencia en algunos casos y falta de formación la mayoría de las veces, sitúan a las personas contratadas de forma temporal en una situación de dificultad para enfrentarse a los riesgos del puesto de trabajo. No se posibilita que exista un conocimiento sobre las medidas preventivas con las que se cuenta en la empresa, si éstas existen, ni por tanto que puedan aplicarse de forma efectiva.

En la mayoría de los casos, las personas jóvenes acceden al mercado de trabajo en una situación de vulnerabilidad, y es esta vulnerabilidad la que está también en relación con condiciones laborales que pueden ocasionar accidentes de trabajo en ese colectivo de edad. Los contratos temporales y la falta de antigüedad que proporcione experiencia contribuyen a la generación de accidentes de trabajo. No en vano, las personas trabajadoras con menos de un ańo de antigüedad en la empresa refieren el doble de accidentes de trabajo que las personas que llevan de uno a tres años en la empresa. Es estructural en nuestro mercado de trabajo «una relación inversa entre antigüedad en el centro de trabajo y accidentabilidad laboral» (Payá, 2019: 129).

La contratación temporal es por todo ello un factor que influye en los accidentes de trabajo. En el conjunto del estado, según el estudio llevado a cabo por un equipo de la Universidad Pompeu Fabra, el riesgo de tener un accidente laboral es 2,5 veces superior para las personas con contrato temporal que para las personas con contrato indefinido. Por otro lado, entre las personas menores de 19 ańos, es tres veces superior la probabilidad de tener un accidente laboral si se tiene un contrato temporal que si se tiene un contrato indefinido (Amable et al., 2001: 176).

Si nos circunscribimos a un plazo de tiempo concreto, entre 2012, año en el que el gobierno aprueba una reforma laboral que es lesiva para los derechos de las personas trabajadoras, y 2018, existe un incremento del número de accidentes de trabajo en las formas de contratación asociadas a la precariedad laboral. El incremento del índice de incidencia en los contratos temporales, que es de un 28 ' $1 \%$, prácticamente quintuplica el incremento que se produce entre las personas que tienen un contrato indefinido, que es de un 6⿳4\%. Pero además, son los 
contratos temporales que también son a tiempo parcial aquellos en los que más se incrementa la siniestralidad, que aumenta un 33’3\% (Chulvi, 2019).

La precariedad laboral genera igualmente riesgos psicosociales, implicando de por sí daños que afectan a la salud mental y psicológica (López Cabrera, 2015: 346). La temporalidad y la inseguridad existente en torno a todo lo que no sea un contrato indefinido a tiempo completo acaban influyendo en el marco psicosocial del puesto en su conjunto. Esto se deriva de la indefensión y la vulnerabilidad que experimentan las personas trabajadoras con contratos precarios. Existen más dificultades para que la persona trabajadora elabore una estrategia defensiva frente a unas malas condiciones de trabajo si tiene un contrato temporal o poca antigüedad, frente a las personas contratadas de forma indefinida y que lleven más tiempo en la empresa.

Prolongación de jornadas de trabajo más allá de lo establecido en el contrato, independientemente de que éste sea temporal o indefinido, y por lo tanto más allá también de lo planificado por la persona como tiempo de trabajo a efectos de la conciliación de la vida laboral y familiar, generan riesgo psicosocial. Y este riesgo además se ve agravado por la desaparición, en algunos casos, de la solidaridad que se haya generado en el grupo de personas trabajadoras. Vogel (2002), en su análisis sobre las condiciones de trabajo en los centros alemanes de investigación y servicios comerciales de la empresa IBM, afirma que el tiempo de trabajo se supedita a las necesidades de producción y de la flexibilidad que sea necesaria para ello. El resultado es que «los trabajadores que no aguantan ese ritmo son rechazados por el grupo y terminan abandonando la empresa» (Vogel, 2002: 99). A aquellas personas que tengan que conciliar vida laboral y familiar, sus propias compañeras y compañeros dejarán de considerarles parte del proyecto.

La necesidad de conciliación entre la vida laboral y familiar es un fenómeno que afecta en mayor medida a las mujeres trabajadoras. La doble presencia o la doble jornada, en la empresa y en casa, es causa también del incremento de riesgos psicosociales. La doble presencia, por lo menos la vinculada al ámbito de los cuidados, entrańa exigencias simultáneas tanto en el trabajo como en la atención a familiares. Ambas exigencias no sólo se dan a la vez, sino que además tienen delimitaciones temporales estrictas. Por ejemplo, si es necesario recoger a un hijo o hija que sale del colegio, es a una hora concreta.

Las mujeres trabajadoras son además un colectivo en el cual los riesgos psicosociales afectan especialmente si se comparan las condiciones de trabajo con las del colectivo de hombres trabajadores. El hecho de que existan menos mujeres trabajadoras en puestos directivos y más en puestos de ejecución, implica que el conjunto de mujeres trabajadoras tienen menos autonomía en sus puestos de trabajo si se compara con el colectivo de hombres trabajadores. Por esto mismo se generan también agravios comparativos entre mujeres y hombres. En el caso 
de poder emprender un desarrollo profesional, la media de las mujeres trabajadoras comenzaría su carrera profesional ascendente (se entiende el sentido ascendente como la trayectoria profesional deseable) desde puestos inferiores que la media de los hombres trabajadores.

La brecha salarial, que afecta a las mujeres y que hace que éstas cobren menos salario que los hombres por realizar el mismo trabajo, no sólo tiene un trasfondo de injusticia en lo referido a la retribución económica. Implica además una falta de reconocimiento del trabajo realizado, y esta falta de reconocimiento es causa de riesgo psicosocial. Y todo ello en un contexto en el que, como recuerda Piketty (2014: 280), en la mayoría de los países las mujeres trabajadoras están «masivamente sobrerrepresentadas dentro del $50 \%$ de las salarios más bajos».

En el conjunto del estado el escenario es semejante. El riesgo de acercarse a la exclusión social es mayor para las mujeres que para los hombres. Esta posibilidad, que también incide en la generación de dolencias de tipo psicosocial, se relaciona directamente con el mercado de trabajo. La posibilidad de tener un empleo que no saque de la pobreza a una persona afecta más a las mujeres que a los hombres, debido a que las primeras acceden en mayor medida que los segundos a "espacios de ocupación vulnerables desde la óptica de los ingresos» (Vicent, 2018: 257).

En un ámbito más cercano, como es el de la CAV, las diferencias salariales y la brecha de género en el mercado laboral también existen. Tal y como han concluido Larrañaga y Martínez (2017: 243) gran parte de la diferenciación viene originada por el hecho de que «el empleo femenino no encaja con el modelo laboral predominante de carrera laboral sin interrupciones y dedicación plena al trabajo remunerado». Los hombres, no obstante, encajan en mayor medida en las carreras laborales sin interrupciones y en la dedicación plena al trabajo remunerado.

Lo analizado hasta ahora es la forma en la que la precariedad laboral influye en los daños a la salud derivados del trabajo, y los mecanismos concretos de influencia mediante los que estos se producen. Todo ello puede verse incluso agravado si se dan otras realidades características del actual modelo de gestión empresarial. Se analiza a continuación cómo la subcontratación es uno de estos factores.

\subsection{Subcontratación y siniestralidad laboral}

La subcontratación de obras y servicios por parte de una empresa principal a otra u otras empresas es un modelo que se encuentra detrás de algunos accidentes de trabajo. Que una empresa recurra a otra para la realización de un trabajo, si bien puede parecer necesario y adecuado en un determinado momento si la empresa principal no dispone entre su plantilla de personas preparadas o formadas para la realización de tareas muy puntuales, concretas y específicas, tareas 
que además puedan incluso no estar directamente relacionadas con la actividad principal de la empresa, implica que las empresas que aspiran a prestar la obra o servicio van a competir entre sí. Las empresas van a querer hacer sus ofertas más atractivas, lo que un modelo de gestión empresarial cortoplacista entiende como más baratas. Esto conlleva reducir costes, degradando y precarizando las condiciones laborales. Los recortes y los ahorros pueden venir incluso de suprimir, retirar o no implantar medidas preventivas encaminadas a que no se generen daños a la salud de las personas trabajadoras.

Existe un modelo claramente abusivo de subcontratación, como es el que se da cuando una empresa principal subcontrata con otra empresa actividades que son parte intrínseca del proceso productivo o que constituyen la propia actividad de la empresa principal. En estos casos el objetivo es el ahorro de costes económicos por parte de la empresa principal. Este ahorro implica condiciones laborales más precarias para las personas trabajadoras.

Hay casos que ilustran esta realidad, por ejemplo accidentes de trabajo mortales ocurridos en empresas de Bizkaia a lo largo de 2020, como el ocurrido en febrero en Astilleros Zamakona o el ocurrido en septiembre en las instalaciones de Derivados del Flúor, empresa de Castro Urdiales, pero en el que falleció un trabajador de la empresa Sisteinge (empresa de Bizkaia). En ambos casos los trabajadores fallecidos llevaban más de diez años trabajando en las instalaciones de las mismas empresas principales, perteneciendo en todo momento a una empresa subcontratada. Alguien que lleva esa cantidad de años trabajando en las mismas instalaciones de una empresa no está realizando una tarea puntual ajena a la actividad propia de la empresa principal, sino que está realizando una actividad que es parte intrínseca de la naturaleza de la empresa principal.

Además de precariedad laboral, existe un agravio comparativo evidente, puesto que lo que se acaba generando es una fragmentación en el colectivo de personas que trabaja en una empresa. Dos personas realizando el mismo trabajo y estando al lado la una de la otra no van a tener el mismo estatus en la empresa y esto afecta a la proyección de futuro en el trabajo y a la posibilidad de carrera profesional (Zubiri, 2011: 85). Como antes se ha expuesto al hablar de la brecha salarial que sufren las mujeres trabajadoras, la falta de reconocimiento y los agravios comparativos influyen también en la generación de riesgos de tipo psicosocial.

La relación existente entre subcontratación y precariedad laboral se incrementa cuando se dan las denominadas cadenas de subcontratación, típicas, por ejemplo, en el sector de la construcción. La empresa principal subcontrata una obra o servicio a otra empresa, y ésta segunda a su vez se lo subcontrata a una tercera que realiza finalmente el trabajo, o que no lo realiza y lo subcontrata a una cuarta. Así sucesivamente, generándose una cadena de empresas subcontratadas con un número determinado de eslabones en cada caso concreto. 
Las cadenas de subcontratación dificultan la coordinación de actividades empresariales para la prevención de riesgos laborales. Esta obligación empresarial afecta a los centros de trabajo en los que concurran personas trabajadoras de dos o más empresas, y viene establecida en el artículo 24 de la Ley 31/1995, de Prevención de Riesgos Laborales. Este precepto legal establece que las empresas han de organizar medios de coordinación para prevenir los riesgos laborales que se den en el centro de trabajo, destacándose la creación de canales adecuados de información entre las empresas y las personas trabajadoras de las mismas y la vigilancia por parte de las empresas principales del cumplimiento por parte de las empresas subcontratadas de la normativa de prevención de riesgos laborales.

Los posibles mecanismos de coordinación vienen desarrollados en el Real Decreto 171/2004, de 30 de enero, por el que se desarrolla el artículo 24 de la Ley 31/1995, de 8 de noviembre, de Prevención de Riesgos Laborales, en materia de coordinación de actividades empresariales. Estos mecanismos han de ser reales, efectivos y contar con la información de la representación especializada de la plantilla en materia de prevención de riesgos laborales. La Inspección de Trabajo y Seguridad Social de Bizkaia se ha posicionado también sobre este asunto a través de un informe originado ${ }^{2}$ tras la denuncia interpuesta por el sindicato CCOO para esclarecer el accidente laboral sufrido por una trabajadora del BBVA. Se establece de forma textual que «no cabe considerar que la coordinación de actividades empresariales en materia preventiva, pueda quedar limitada a la mera información recíproca». No es suficiente con que las empresas concurrentes en un centro de trabajo se limiten a intercambiar las evaluaciones de riesgos, por ejemplo.

Cuando en un mismo centro de trabajo, o en un mismo colectivo de personas trabajadoras se entrecruzan la subcontratación con otras formas de precariedad laboral, como es la temporalidad en la contratación o la prolongación de horarios de trabajo más allá de lo pactado, existen aún más factores que puedan propiciar accidentes de trabajo. La negociación colectiva puede, como se expone a continuación, generar mecanismos que ayuden a paliar esta situación.

\section{La negociación colectiva como instrumento de mejora de la salud laboral}

\subsection{La negociación colectiva como garantía de derechos}

El derecho del trabajo tiene su origen en el hecho de que en las relaciones laborales existe una relación de subordinación. En la relación hay una parte de por sí más vulnerable, que es la persona trabajadora, y el derecho del trabajo tiene la función de tutelar las condiciones de esa parte más vulnerable.

2 Inspección de Trabajo y Seguridad Social de Bizkaia (2019) Informe de la de orden de servicio 48/00009056/18. 
La negociación colectiva, desde el punto de vista de las personas trabajadoras y de quienes las representan, tiene el mismo objetivo, y en la actualidad tiene también que hacer frente a una nueva concepción del derecho del trabajo, defendida desde ámbitos políticos liberales, que pretende que el objetivo del derecho del trabajo se supedite a la creación de empleo (Bilbao, 1999-1: 81), y cuando hablamos en este contexto de creación de empleo, lo hacemos desde un punto de vista cuantitativo. Vale cualquier tipo de empleo que pueda pasar a formar parte de las estadísticas como empleo creado.

La defensa de unas condiciones de trabajo dignas y la tutela de la persona trabajadora pasan para este nuevo derecho del trabajo a un segundo plano. De ahí la importancia de la negociación colectiva, como instrumento para hacer frente y contrarrestar este nuevo paradigma. También desde el punto de vista de la salud laboral.

La negociación colectiva es un instrumento necesario para evitar que las relaciones laborales se individualicen. La ausencia de negociación colectiva crea un escenario propicio para que surjan lo que Recio (2004) denomina «nuevas pautas de gestión de personal orientadas a individualizar y generar presión sobre el comportamiento de cada empleado». El instrumento de defensa y generación de derechos colectivos desaparece, y las empresas, de forma ya individualizada, pueden generar e implantar condiciones de trabajo más precarias, que acaban afectando como ya se ha explicado a la salud de las personas.

La reforma laboral impulsada por el gobierno del Partido Popular en el año 2012 tenía entre sus objetivos desarticular la negociación colectiva. Se le otorgaba prevalencia aplicativa al convenio de empresa frente al convenio provincial sectorial, lo que acaba por reducir el tamaño del ámbito de negociación y circunscribirlo al centro de trabajo. Como nos dice Laurent Garrouste, «en la ofensiva capitalista contra el derecho del trabajo, la voluntad de centrar la negociación colectiva a nivel de empresa constituye una orientación fundamental» (Garrouste, 2014: 44). No en vano, y por poner un ejemplo, uno de los compromisos que el Consejo de la UE exigió al gobierno griego en 2011 para la concesión de ayudas financieras fue la suspensión de la aplicación de convenios sectoriales, argumentando que facilitaba la adaptación de los salarios en función de las condiciones económicas.

Una vez definida la negociación colectiva como instrumento que mejora las condiciones de trabajo de las personas, veremos la posibilidad de que se generen cláusulas concretas que ayuden a la defensa de la salud laboral.

\subsection{Participación de las personas trabajadoras en materia de seguridad y salud en el trabajo}

La participación de las personas trabajadoras en materia preventiva en el seno de la empresa es una de las mejores garantías que pueden existir para la im- 
plantación de medidas frente a los daños a la salud derivados del trabajo. No en vano, la propia Ley 31/1995, de Prevención de Riesgos Laborales, desarrolla esta participación en todo su capítulo $\mathrm{V}$, y otorga un protagonismo especial a las delegadas y delegados de prevención a través de los cuales se canaliza fundamentalmente la citada participación.

Para valorar la importancia de esta figura hay que resaltar las facultades de paralización del puesto de trabajo por riesgo grave e inminente que le otorga el artículo 21 de la propia Ley 31/1995, de Prevención de Riesgos Laborales. Si bien en este artículo se dice que las propias personas trabajadoras también pueden llevar a cabo la paralización por riesgo grave e inminente, la práctica real, cuando se dan estas circunstancias, muestra que donde hay delegadas y delegados de prevención se encargan de llevar a cabo esta paralización. Donde no existe la representación especializada en esta materia, por el contrario, no suele darse este tipo de paralizaciones de puesto de trabajo.

Esto influye en el hecho de que haya menos daños a la salud en el trabajo en las empresas en las que hay representación especializada en materia de prevención de riesgos laborales. En un estudio que abarca los países de la Unión Europea, Payá y Beneyto (2019) han llegado a la conclusión de que allí donde existe una legislación más avanzada en materia de participación y con mayor cobertura de las personas en la negociación colectiva, se da un índice de accidentes inferior a un $30 \%$ de la media. La conclusión es que «la intervención de los sindicatos influye positivamente en la seguridad y salud laboral» (Payá y Beneyto, 2019: 46)

Es posible, desde la negociación colectiva, fomentar mejoras en la participación de las delegadas y delegados de prevención, sobre todo si tenemos en cuenta que la Ley 31/1995, de Prevención de Riesgos Laborales, remite de forma concreta a ello en su artículo 35. En relación a la designación de delegadas y delegados de prevención, más allá de la designación clásica entre quienes ya son representantes de la plantilla, existe la posibilidad de articular mecanismos de elección que garanticen que la facultad corresponde a las y los representantes del personal o a las personas trabajadoras. Tales mecanismos han de recogerse en el convenio colectivo de aplicación.

Este mismo precepto legal regula también la necesaria existencia en el convenio colectivo de cláusulas sobre la posible creación de órganos que asuman las competencias de las delegadas y delegados de prevención en todos los centros de trabajo en los que se aplique ese convenio colectivo. Esto es de especial interés en la CAV, con un tejido empresarial donde abundan las microempresas, en las que si no hay un mínimo de seis personas trabajadoras no se podrán realizar elecciones sindicales para la elección de representantes de personal.

Una de las facultades fundamentales de las delegadas y delegados de prevención, junto con el control y vigilancia del cumplimiento de la aplicación de me- 
didas preventivas y del cumplimiento de la legislación por parte de la empresa, es la colaboración en la realización de la evaluación de riesgos. Si bien la realización de la evaluación de riesgos atañe, como obligación legal, exclusivamente a la empresa, el artículo 36.2.a) de la Ley 31/1995, de Prevención de Riesgos Laborales, establece que es una facultad de las delegadas y delegados de prevención acompañar al personal técnico que realiza las citadas evaluaciones. El objetivo es que se aporte la visión de las personas trabajadoras, y no sólo el punto de vista técnico. Cabe recordar que la evaluación de riesgos es un documento de gran importancia, entre otras cosas porque de ella se deriva la planificación de la actividad preventiva.

El artículo 4.2 del Real Decreto 39/1997, de 17 de enero, por el que se aprueba el Reglamento de los Servicios de Prevención, establece una serie de supuestos en los cuales deberá de revisarse la evaluación de riesgos, como cuando haya cualquier cambio en las condiciones de trabajo, se modifiquen procedimientos o cuando se incorporen personas trabajadoras especialmente sensibles a las condiciones de un puesto de trabajo. Más allá de esto, y no produciéndose estas circunstancias, no existe obligación de revisar la evaluación de riesgos por parte de la empresa. No existe un plazo temporal para revisar la evaluación.

Es aquí donde radica la importancia de la negociación colectiva, y su reflejo en los convenios, estableciendo por ejemplo revisiones que han de darse en plazos concretos de tiempo. Esto garantiza una actualización constante del conocimiento sobre los riesgos, una atención más continua sobre los mismos, la extensión de la cultura preventiva y el seguimiento sobre la implantación de medidas. De estas cláusulas, en términos de prevención, se benefician también personas trabajadoras recién incorporadas a la empresa o pertenecientes a empresas subcontratadas.

Asimismo, mediante la negociación colectiva ha sido posible establecer en determinados convenios cláusulas que incrementen el nivel de protección de las personas trabajadoras de empresas subcontratadas o de otras figuras no pertenecientes a la empresa principal, como las personas de empresas de trabajo temporal. Según recoge el estudio ${ }^{3}$ del Instituto Sindical de Trabajo, Ambiente y Salud (Uberti-Bona et al., 2003: 201) en el Convenio de la Construcción de Navarra hay cláusulas que permiten a las personas trabajadoras de ETT participar en materia preventiva a través de los órganos para tal fin constituidos en la empresa usuaria. Es un ejemplo extensible a las plantillas de las empresas subcontratadas.

${ }^{3}$ Es recomendable la lectura del estudio referido, puesto que se trata de una extensa recopilación de cláusulas existentes, o que al menos lo fueron en un momento dado, en gran número de convenios colectivos. En el estudio se hace especial hincapié lo referido a participación y representación de las personas trabajadoras. 
El Convenio Colectivo de la Industria Siderometalúrgica de Bizkaia ${ }^{4}$ incluye un artículo específico sobre salud laboral, concretamente el 32, que aborda aspectos relevantes. En materia de subcontratación y coordinación de actividades empresariales para la prevención de riesgos laborales, se mantiene una cláusula que figuraba en anteriores convenios, estableciéndose de forma específica que la persona que es delegada de prevención en la empresa subcontratada puede acceder al centro de trabajo de la empresa principal para la comprobación in situ de las condiciones de trabajo. Las personas que son delegadas de prevención también podrán solicitar que se realicen reuniones para la valoración de los medios de coordinación establecidos. Por otra parte, hay una novedad importante, como es la delegación en la comisión mixta del convenio de la creación de una figura denominada "Asistente sectorial de prevención», con posibilidad de intervenir ante casos de accidentes graves o muy graves. Esta figura tiene gran relevancia, puesto que está facultada también para intervenir en empresas en las que no exista representación sindical, como en el caso del gran número de microempresas que hay en el ámbito de aplicación de este convenio.

Participación real y efectiva de las personas trabajadoras en la prevención de riesgos laborales, existencia de delegadas y delegados de prevención e interlocución con la empresa en la implantación de medidas preventivas son aspectos que mejoran la salud laboral y se deben reforzar en las empresas. Pero más allá del centro de trabajo o de la relación laboral, existe otro espacio para mejorar la salud laboral: incidir sobre la gestión de las contingencias profesionales y comunes a través de las mutuas.

\subsection{Las mutuas y su incidencia en la gestión de la salud laboral}

Existe la posibilidad también de abordar en la negociación colectiva aspectos relativos a las problemáticas que la gestión de las contingencias profesionales, y en muchos casos también de las comunes (enfermedad común y accidente no laboral), por parte de las mutuas ocasiona a las personas trabajadoras. La colaboración de las mutuas en el pago de la prestación de incapacidad transitoria (de aquí en adelante, IT) por contingencias comunes, que pueden llevar a cabo desde 1995, faculta a estas entidades colaboradoras con la Seguridad Social para presionar más a las personas de IT por esta contingencia. Esto puede ocurrir en todas aquellas empresas que hayan dejado la gestión de la contingencia común en manos de las mutuas.

Las mutuas pueden citar de forma arbitraria a las personas trabajadoras en situación de IT por contingencias comunes y, si bien no pueden emitir el alta

${ }^{4}$ Convenio colectivo provincial del Sector de la Industria Siderometalúrgica de Bizkaia 2019-2021, Disponible en: https://convenioscolectivos.net/industria-siderometalurgica-de-bizkaia/ (Accedido: 5-10-2020) 
médica, ya que esta competencia es exclusiva del sistema público de salud, pueden formular propuestas de alta. El artículo 6.2 del Real Decreto 625/2014, de 18 de julio, por el que se regulan determinados aspectos de la gestión y control de los procesos por incapacidad temporal en los primeros trescientos sesenta y cinco días de su duración, redujo a cinco días el plazo con el que contaba el sistema público de salud para contestar a las propuestas de alta de la mutua.

Las personas que están en situación de IT por contingencia común tienen que presentarse a la citación de la mutua correspondiente, y si no se presentasen, la mutua, que es quien gestiona y paga su prestación económica por IT, puede extinguir el pago de la prestación. Esto es un mecanismo de presión de las mutuas hacia las personas que estén en situación de IT por contingencias comunes, porque el hecho de que la legislación no se pronuncie sobre la periodicidad de esas citas a revisión, da lugar a frecuentes abusos por parte de las mutuas.

Este tipo de prácticas por parte de las mutuas se da en las empresas en las que éstas cedieron a aquellas la gestión de la contingencia común. Para poner freno a esto es necesario introducir en los convenios colectivos el compromiso de la empresa de que las contingencias comunes no serán gestionadas por las mutuas, sino que su gestión permanecerá en la Seguridad Social, así como la reversión de este hecho en aquellas empresas que ya hayan cedido la gestión de la contingencia común a la mutua. Se evita así que las personas enfermas en situación de IT por contingencias comunes acaben solicitando el alta médica de forma voluntaria al sistema público de salud, solicitud que en caso de materializarse va en contra de su proceso de recuperación.

La negociación colectiva también permite abordar el establecimiento de indicadores de calidad en el servicio para que la empresa renueve o no el documento de adhesión con la mutua, transcurrido el año natural que dura la vigencia de la adhesión. La empresa es la que elige renovar o no la adhesión con una mutua concreta. Por ello, existen elementos que la representación legal de la plantilla puede plantear a la empresa, que es quien elige la adhesión a la mutua y que puede por tanto evitar o propiciar conflictos en este sentido. Estos indicadores son los siguientes:

- Accesibilidad para los trámites administrativos.

- Información que se da a la persona trabajadora cuando acude a revisión.

- Trabas a la compensación del desplazamiento al lugar en el que se hacen las revisiones médicas en la IT por contingencia común.

- Acceso a la hoja de reclamaciones a quien lo solicite.

- Observancia estricta de las normas de confidencialidad de datos.

- Existencia o no de una comunicación motivada, por escrito y fehaciente previa a las citaciones.

— Calidad y accesibilidad de las instalaciones. 
— Existencia o no de una persona interlocutora en la mutua para la resolución rápida de dudas.

- Satisfacción percibida y expresada por las personas usuarias.

Queda también espacio para cláusulas relativas a la codecisión en la elección de la mutua. La elección de qué mutua va a asegurar las contingencias debería ser algo acordado entre plantilla y empresa, y no una elección de la empresa. En este momento, en ausencia de estas cláusulas en los convenios, es una decisión unilateral de la empresa.

El argumento empresarial habitual de que es la empresa quien abona la cotización de las contingencias profesionales de la plantilla, y por tanto ha de elegir la mutua, no se sustenta, puesto que el asegurado es un tercero, la persona trabajadora en este caso. Quien puede acabar sufriendo una mala praxis médica de la mutua y los abusos a los que nos hemos referido en los párrafos anteriores, también es la persona trabajadora.

La codecisión se aborda, constituyendo un buen ejemplo, en el Convenio Colectivo de la Construcción de Álava ${ }^{5}$, que establece en su Disposición Adicional Segunda que la empresa que quiera cambiar de mutua, tendrá que requerir informe previo de la representación legal de la plantilla, el cual deberá ser emitido en el plazo de 15 días, y que si la citada representación legal no lo emite en ese plazo, el informe se entenderá emitido favorablemente. Si este informe fuese negativo o en votación secreta adoptada por dos tercios de la plantilla, se formulase propuesta de cambio de mutua, la empresa deberá remitir a la Comisión Paritaria del Convenio un escrito dando cuenta de la propuesta, comprometiéndose ambas partes a acudir a un procedimiento de resolución de conflicto (PRECO).

Es imprescindible el refuerzo de la participación de la plantilla y sus representantes también en la relación de una empresa con su mutua correspondiente. La negociación colectiva puede, en este sentido, contribuir a garantizar una mejor recuperación de las personas que están en situación de IT. No obstante, existe un problema: la negociación colectiva no llega a todas las personas trabajadoras.

\subsection{El reto de incluir plataformas digitales y falsos autónomos en la negociación colectiva}

La proliferación de la economía basada en plataformas digitales es también en el presente una característica del mercado del trabajo, y es algo que está configurando unas nuevas relaciones laborales. Si bien la producción bajo demanda,

5 Convenio colectivo para el sector de la construcción y obras públicas de Álava 2017-2020, Disponible en: https://www.cnc.es/cc/ccalava.pdf (Accedido: 6-10-2020) 
entendida como no producir bienes o servicios que no hayan sido previamente requeridos por la persona que se quiere hacer con ellos, ya existía, en esta nueva etapa hay una intensificación de este modo de producción. La generación de plataformas digitales que ponen en contacto directamente a la persona prestadora de un servicio con la persona usuaria final es la generación para una sola persona trabajadora de «una multiplicidad de contratos de trabajo, de duración irrisoria, con multitud de empresarios» (Todolí, 2017: 169). Pero es también una huida hacia adelante de empresas que pretenden cumplir simplemente un papel de intermediación, pero que se sirven de las plataformas digitales que han generado para que en la relación con la persona que presta el servicio no se aplique el derecho del trabajo ni exista la negociación colectiva. El conflicto de intereses que existe entre la persona que presta el servicio y la plataforma digital es el mismo que entre persona trabajadora y la empresa en una relación laboral. Solo cambia la forma, en un intento de eludir el derecho del trabajo.

Se construye así una relación en la que surge una figura que no es nueva: la persona que es falso autónomo. En este marco, como es obvio, no hay delegados ni delegadas de prevención debido a que «no reconociendo la relación laboral de las personas que trabajan para ellas, lo primero que limitan estas plataformas es la participación y la representación» (Ríos, 2020). Grave perjuicio, puesto que estas plataformas digitales generan riesgos psicosociales importantes, que derivan de que las personas que prestan el servicio son valoradas por un algoritmo que toma sus propias decisiones y ante el que no se puede protestar ni presentar argumentos. Además, la valoración que las personas usuarias hacen de quien presta el servicio, y que es gestionada de forma directa por el algoritmo, ha ocasionado por ejemplo accidentes de tráfico entre las personas conocidas como «riders», que prestan servicios en plataformas digitales de reparto de comida a domicilio, debido a la necesidad de entregar el pedido lo antes posible para que el algoritmo no les penalice por posibles malas opiniones de las personas usuarias.

Una sentencia del Tribunal Supremo ${ }^{6}$ establece que las personas que prestan este tipo de servicios son falsas autónomas, y que tienen que regirse por una relación laboral al uso, como es la del régimen general de la Seguridad Social. En el trabajo que se presta a través de plataformas digitales hay aspectos que hacen que esto sea evidente, como que no sea el cliente final quien abone el servicio a quien lo presta, sino la plataforma, que fija igualmente la forma de pago.

Las plataformas digitales no son meras intermediarias como pretenden, sino que son empresas, que organizan la prestación del servicio como otra empresa cualquiera. Como empresas deben aplicar un convenio colectivo, abriéndose las

6 Sentencia del Tribunal Supremo STS 2924/2020, de 25 de septiembre (n. ${ }^{\circ}$ de recurso 4746/2019) Disponible en: http://www.poderjudicial.es/search/openDocument/05986cd385feff03 (Accedido: 3-12-2020) 
posibilidades de acción en la negociación colectiva. Desde el punto de vista de la salud laboral la prioridad es, por lo tanto, la organización de las personas trabajadoras en el seno de esas empresas, y que accedan a los derechos de representación y participación a los que hemos aludido anteriormente.

El problema de las personas que desempeñan su tarea como falsas autónomas en relación a la prevención de riesgos laborales no es nuevo. En sectores como el de la construcción prolifera esta figura, que además puede estar inmersa en las cadenas de subcontratación a las que antes nos hemos referido. Esto es anterior a la existencia de las propias plataformas digitales. Los falsos autónomos y falsas autónomas rara vez van a interpelar a la empresa principal para la que trabajan debido a una posible falta de medidas preventivas, y es impensable que se acojan a la paralización del puesto de trabajo por riesgo grave e inminente, a la que hemos aludido en la explicación del artículo 21 de la Ley 31/1995, de Prevención de Riesgos Laborales, puesto que esto implicaría la rescisión del contrato por parte de la empresa usuaria para la cual están trabajando (Ríos, 2013: 141). Hablamos, como se ve, y desde hace tiempo, de una figura vulnerable, que la expansión de las plataformas digitales no ha hecho sino extender. Si bien anteriormente, y debido a la naturaleza de la relación existente entre la empresa principal y la persona falsa autónoma, era difícil la intervención de la negociación colectiva para la mejora de las condiciones de trabajo, la sentencia del Tribunal Supremo referida abre una vía para que esta problemática pueda regularse mínimamente, por lo menos en el marco de las plataformas digitales.

La extensión de la negociación colectiva de forma real y efectiva a estos grupos de personas, contribuiría a mejorar sus condiciones de trabajo y también su salud laboral, porque la negociación colectiva sería un freno a la extensión de la precariedad laboral.

\section{Conclusiones}

Tras revisar las diferentes realidades que existen en torno a la precariedad laboral, se ha podido demostrar que en multitud de ocasiones este fenómeno es causa directa de daños a la salud derivados de las condiciones de trabajo, y que no está circunscrito sólo a la siniestralidad laboral, hecho que se relaciona de forma directa con la temporalidad en la contratación y con la subcontratación de obras y servicios, sino que también influye en la generación de riesgos psicosociales. Además, esta última tipología de riesgos ha de analizarse bajo el punto de vista de género, pues afecta en mayor medida a las mujeres trabajadoras.

La negociación colectiva es un instrumento que para las personas trabajadoras y sus representantes tiene el objetivo de generar derechos que afecten a un número de personas determinado. La conclusión que se extrae tras el análisis 
realizado es que para mejorar la salud de las personas y minimizar la existencia de riesgos en el trabajo, es necesario potenciar la participación de las personas trabajadoras.

En el centro de trabajo, la participación se da fundamentalmente a través de las delegadas y delegados de prevención. La legislación establece que esta figura, fundamental e insustituible, tiene un papel importante para canalizar demandas, tanto en el seno de la propia empresa, como hacia otro tipo de entidades, por ejemplo, la propia Inspección de Trabajo y Seguridad Social. Para la defensa de la salud laboral de las personas trabajadoras, uno de los objetivos de la negociación colectiva pasa por fortalecer esta figura, que puede incidir directamente en la mejora de las condiciones de trabajo del personal subcontratado y en la implantación de medidas preventivas para que no se generen daños a la salud en el trabajo.

Como también se ha expuesto, la participación y la negociación colectiva pueden ayudar a establecer ciertas limitaciones a actuaciones abusivas de la mutua. Hablamos básicamente del control al que las mutuas someten a las personas en situación de IT en caso de contingencias comunes, y que en ocasiones constituyen una injerencia en el proceso de recuperación de una dolencia.

Se ha demostrado asimismo que la generación de derechos, mediante la negociación colectiva, debe alcanzar a las personas prestadoras de servicios a través de plataformas digitales y a aquellas que son falsas autónomas, para contribuir a ordenar las relaciones laborales y a eliminar el dumping social entre personas trabajadoras.

La negociación colectiva, por todo ello, es imprescindible para que se regulen y establezcan unas condiciones de trabajo iguales para todo el colectivo afectado por un convenio colectivo concreto, y no existan personas trabajadoras que se vean en la necesidad u obligación de aceptar unas condiciones de trabajo que sean inferiores a las de otra. Hablamos pues de un instrumento encaminado a poner límite a la precariedad laboral.

En ausencia de negociación colectiva, las relaciones entre empresa y persona trabajadora se individualizan. En este escenario, la parte vulnerable, que es la persona trabajadora, acaba perdiendo derechos y viendo sus condiciones laborales degradadas y precarizadas, lo que se relaciona con la aparición de accidentes y de daños a la salud derivados de las condiciones de trabajo.

La demanda de las personas trabajadoras y el objetivo de sus representantes en la negociación colectiva es el empleo de calidad, con unas condiciones de trabajo que no degraden la vida de las personas. El sociólogo Andrés Bilbao lo explica perfectamente: «aunque nos haga mucha falta un empleo, si sólo pedimos trabajo, lo que estamos pidiendo es un explotador que nos robe la vida» (Bilbao, 1999-2: 12) 
Un mercado de trabajo sin precariedad laboral es un objetivo a conseguir, y conlleva un escenario con menos accidentes de trabajo y menos enfermedades profesionales. Es, por tanto, la mejor garantía para el respeto de los derechos de las personas trabajadoras.

\section{Bibliografía}

Amable, M., Benach, J. y González, S (2001) «La precariedad laboral y su repercusión sobre la salud: conceptos y resultados preliminares de un estudio multimétotodos», Archivos de Prevención de Riesgos Laborales, 4, 169-184. Disponible en: https://archivosdeprevencion.eu/view_document.php?tpd=2\&i=1281 (Accedido: 24-9-2020)

Bilbao, Andrés (1999-1) «La nueva regulación del mercado de trabajo en España», en Prieto, Carlos (ed.), La crisis del empleo en Europa (Volumen 1), Valencia: Germania, 65-82.

Bilbao, Andrés (1999-2) El empleo precario. Seguridad de la economía e inseguridad del trabajo, Madrid: Los Libros de la Catarata.

CCOO (2007) La prevención de riesgos en los lugares de trabajo. ISTAS

Chulvi, Berta (2019) «Accidentes: la huella más dramática de la precariedad», Por Experiencia, 81. Disponible en: https://porexperiencia.com/condiciones-de-trabajo/accidentes-la-huella-mas-dramatica-de-la-precariedad (Accedido: 20-9-2020)

Convenio colectivo para el sector de la construcción y obras públicas de Álava 2017-2020, Disponible en: https://www.cnc.es/cc/ccalava.pdf (Accedido: 6-10-2020)

Convenio colectivo provincial del Sector de la Industria Siderometalúrgica de Bizkaia 20192021, Disponible en: https://convenioscolectivos.net/industria-siderometalurgica-debizkaia/ (Accedido: 5-10-2020)

Fina, Lluís (2001) «El aumento del trabajo a tiempo parcial en Europa. Problemas y oportunidades», en Lorente Campos, Raúl (ed.), Trabajo a tiempo parcial, Valencia: Germania, 87-94.

Garrouste, Laurent (2014) «La crisis y las condiciones de explotación del trabajo en Europa», Viento Sur, 134, 41-53. Disponible en: https://vientosur.info/wp-content/ uploads/spip/pdf/VS134_L_Garrouste_La_crisis_y_condiciones_de_trabajo_Europa. pdf (Accedido: 19-9-2020)

Larrañaga Sarriegi, Mertxe y Martínez Tola, Elena (2017) «Desigualdades económicas de género en la Comunidad Autónoma del País Vasco», Ekonomiaz, 91, 209-247 Disponible en: file://C:/Users/78889745/Downloads/Dialnet-DesigualdadesEconomicasDeGeneroEnLaComunidadAutono-6038698.pdf (Accedido: 31-10-2020)

Ley 31/1995, de 8 de noviembre, de Prevención de Riesgos Laborales. Disponible en: https:// www.boe.es/eli/es/1/1995/11/08/31 (Accedido: 4-10-2020)

López Cabrera, Aida (2015) «Riesgos psicosociales derivados de la precariedad laboral en la CAV: posibles líneas de actuación", Lan Harremanak, 32, 337-357 Disponible en: https://ojs.ehu.eus/index.php/Lan_Harremanak/article/view/15427/13940 (Accedido: 16-9-2020)

Lorente Campos, Raúl y Guamán Hernández, Adoración (2018) «Expansión de la temporalidad y erosión de la relación de empleo estándar en España. ¿La irrupción de un nuevo paradigma de relación de empleo?», Cuadernos de Relaciones Laborales, 36, 35-63. Disponible en: https://revistas.ucm.es/index.php/CRLA/article/ view/59556/4564456546787 (Accedido: 26-9-2020) 
Payá Castiblanque, Raúl (2019) «Contexto económico y determinantes sociales de la accidentabilidad laboral en el sur de Europa. Los casos portugués y español», e-cadernos CES, 31, 116-146. Disponible en: file://C:/Users/78889745/Downloads/eces4350\%20(1).pdf (Accedido: 25-9-2020)

Payá Castibalnque, Raúl y Beneyto Calatayud, Pere J. (2019) «Representación sindical y prevención de riesgos laborales durante la crisis: impacto y límites», Sociología del Trabajo, 94, 29-62. Disponible en: https://revistas.ucm.es/index.php/STRA/article/ view/63303/4564456549293 (Accedido: 30-9-2020)

Piketry, Thomas (2014) El capital en el sigloXXI, Madrid: Fondo de Cultura Económica

Real Decreto 39/1997, de 17 de enero, por el que se aprueba el Reglamento de los Servicios de Prevención. Disponible en: https://www.boe.es/eli/es/rd/1997/01/17/39 (Accedido: 4-10-2020)

Real Decreto 625/2014, de 18 de julio, por el que se regulan determinados aspectos de la gestión y control de los procesos por incapacidad temporal en los primeros trescientos sesenta y cinco dias de su duración. Disponible en: https://www.boe.es/eli/es/rd/2014/07/18/625 (Accedido:1-10-2020)

Recio, Albert (2004) «¿Qué fue de la clase obrera?», en Mientras Tanto, 93.

Ríos, Alfonso (2013) «Trabajadores autónomos y salud laboral: claves de una problemática», en Riobello, Moisés, El autónomo en la coordinación de prevención en $360 .^{\circ}$, Valladolid: Thomson Reuters Lex Nova, 138-147.

Ríos, Alfonso (2020) «Plataformas digitales y reparto a domicilio: un peligro también para la salud laboral de los riders», Naiz, Disponible en: https:/www.naiz.eus/es/iritzia/articulos/plataformas-digitales-y-reparto-a-domicilio-un-peligro-tambien-para-la-saludlaboral-de-los-riders (Accedido: 5-10-2020)

Todolí, Adrián (2017) «Sobre la llamada economía colaborativa y sus implicaciones laborales y sociales», Gaceta Sindical, 29, 159-182

Uberti-Bona, Valeria, Rodrigo, Fernando, Camacho, Jesus y Briso, Pedro (2003) La Salud Laboral en la Negociación Colectiva en España (1995-2002) ISTAS Disponible en: http://istas.net/descargas/negociacion.pdf (Accedido: 2-10-2020)

Vicent Valverde, Lucía (2018) Precariedad laboral en la economía española. Una perspectiva de género, Tesis doctoral Universidad Complutense de Madrid, Madrid. Disponible en: https://eprints.ucm.es/49822/1/T40505.pdf (Accedido: 27-10-2020)

Vogel, Laurent (2002) «La organización del trabajo: un ámbito decisivo en la lucha contra los sufrimientos psicológicos», Lan Harremanak, Número especial, 93-101. Disponible en https://ojs.ehu.eus/index.php/Lan_Harremanak/article/view/5868/5544 (Accedido: 26-9-2020)

Zubiri Rey, Jon Bernat (2011) «Precariedad, movimientos de huelga y sindicalismo en Francia», Encrucijadas. Revista Critica de Ciencias Sociales, 1, 70-90. Disponible en: file://C:/Users/78889745/Downloads/Precariedad_movimientos_de_huelga_y_ sindicalismo_e.pdf (Accedido: 3-10-2020) 